\title{
Evaluating neoclassical tearing mode detection with ECE for control on ITER
}

\author{
H. van den Brand ${ }^{1,2,3}$, M.R. de Baar ${ }^{2,3}$, N.J. Lopes \\ Cardozo $^{1}$ and E. Westerhof ${ }^{3}$ \\ ${ }^{1}$ Fusion Science and Technology Group, Eindhoven University of Technology \\ P.O. Box 513, 5600 MB Eindhoven, The Netherlands \\ 2 Control Systems Technology Group, Eindhoven University of Technology P.O. \\ Box 513, 5600 MB Eindhoven, The Netherlands \\ ${ }^{3}$ FOM Institute DIFFER - Dutch Institute for Fundamental Energy Research, \\ Association EURATOM-FOM, Trilateral Euregio Cluster, P.O. Box 1207, 3430 \\ BE Nieuwegein, The Netherlands \\ E-mail: E.Westerhof@differ.nl
}

\begin{abstract}
Neoclassical Tearing Mode (NTM) control on ITER requires detection of the mode location to be accurate and with low latency. This paper presents a systematic way to evaluate mode detection algorithms for ITER using numerical simulations of Electron Cyclotron Emission (ECE), taking into account the radial asymmetry in the temperature perturbation by a rotating magnetic island. Simulated ECE is detected using a synthetic radiometer, in the ITER equatorial port plug, and processed by two detection algorithms for the $2 / 1$ and $3 / 2$ NTMs for a burning H-mode ITER plasma. One of the algorithms also incorporates simulated Mirnov data. The video bandwidth is set at $2 \mathrm{kHz}$. This allows for intermediate frequency bandwidths of $B_{I F}=400 \mathrm{MHz}$ and $B_{I F}=300 \mathrm{MHz}$ for the two algorithms respectively. The intermediate frequency bandwidth provides a trade-off between radial accuracy (low bandwidth) and low noise/latency (large bandwidth). $2 / 1$ and $3 / 2 \mathrm{NTMs}$, seeded with widths up to $9 \mathrm{~cm}$ and $11 \mathrm{~cm}$, are detectable with the required accuracy within $250 \mathrm{~ms}$. With appropriate settings for the radiometer, the NTM detection using ECE is accurate and with low latency. The algorithm that incorporates both ECE and Mirnov data showed the lowest detection latencies.
\end{abstract}

Keywords: NTM, ECE, detection, latency and ITER

Submitted to: Nuclear Fusion 


\section{Introduction}

The ITER tokamak (major radius $R_{0}=6.2 \mathrm{~m}$, minor radius $a=2 \mathrm{~m}$ ) is being constructed near Cadarache, France.[1] Plasmas in ITER should demonstrate a fusion gain of $Q=10$ for a period of $300 \mathrm{~s}$. Achieving this goal requires control of detrimental instabilities such as the Neoclassical Tearing Mode (NTM), an MHD instability that reduces core temperature and thereby fusion yield. NTMs can result in a sudden loss of plasma (a disruption).[2] NTMs are global magnetic perturbations with poloidal and toroidal mode numbers $m$ and $n$ respectively, leading to reconnection of magnetic field lines forming a magnetic island at the rational flux surface described by the safety factor $q=m / n$. A magnetic island is characterized by its island width $w$, representing the amplitude of the mode, and its toroidal rotation frequency $\omega$. The metastable NTM requires a seed island to become unstable and grows as a result of a lack of local bootstrap current inside the island. This current deficiency can be compensated through Electron Cyclotron Resonance Heating (ECRH) and Current Drive (ECCD).[3] NTMs with mode numbers $m / n=2 / 1$ and $m / n=3 / 2$ will degrade the core temperature of ITER plasmas the most.[4] The presence of a magnetic island can be detected by its magnetic perturbation or the effect of the island on the temperature profile. Control of $2 / 1$ and $3 / 2$ NTMs is required for sustained high performance burning H-mode ITER plasmas and should prevent all NTMs from causing a disruption.

Several strategies exist for NTM control, the most prominent of which deals with full suppression of NTMs using ECCD directly after detection of seeded islands.[4] The Generalized Rutherford Equation (GRE), describing island growth, was used by La Haye et al and Van den Brand et al to assess the required accuracy in the ECCD deposition position, for ITER.[5][6] Full suppression of the 2/1 and 3/2 NTM requires a radial accuracy of the deposition position of $7 \mathrm{~mm}$ and $5 \mathrm{~mm}$, respectively. Due to a decreased efficiency for small island widths, for larger misalignments, ECCD is incapable of fully suppressing NTMs,.[6] The accuracy is expressed in coordinates provided by an average of the minor radius in the equatorial plane on the low and high field side. A control system should achieve the deposition accuracy to be able to fully suppress the NTMs.

Whether a control system would work for ITER, requires an analysis of the accuracy and latency of the system. As a requirement on the control system, the maximum allowed latency is determined, which is the longest allowed time between the seeding of the mode and the start of ECCD that still results in full suppression of the island before mode locking, i.e. stop of mode rotation.[6] The control loop should make the ECCD deposition position converge to the mode position up to the required accuracy within the maximum allowed latency. The maximum allowed latency depends on the seed island width and the evolution of island width and rotation, for which a number of theoretical models with associated parameters is known to give an accurate description of current measurements. Two models for the bootstrap growth rate at small island widths exist: the polarization model, describing the decreasing growth by the ion polarization current induced by the different response of ions and electrons to the island rotation, and the transport model, explaining the decreasing growth by an incomplete flattening of the pressure profile due to a competition of parallel and perpendicular transport. At the marginal island width

$w_{\text {marg }}$, expected between 2 and $6 \mathrm{~cm}$ for ITER [4], the maximum growth rate is found. The maximum allowed latency is smallest, i.e. posing the most stringent 
requirements, for the polarization model with $w_{\text {marg }}=2 \mathrm{~cm}$.[6] This model is used in the remainder of this paper. Using this model, the maximum allowed latency is 1 and 3 seconds for a seed island width of $2 \mathrm{~cm}$ for the $2 / 1$ and $3 / 2 \mathrm{NTM}$, respectively, and becomes zero at seed island widths of $9.5 \mathrm{~cm}$ and $12 \mathrm{~cm}$, respectively.[6] NTMs seeded with a larger width cannot be suppressed before mode-locking and necessitate an alternative control strategy, which uses for instance magnetic perturbation fields, to control locked modes.[7]

In order to reach the required deposition accuracy within the maximum allowed latency, detection should result in an accurate mode location within a fraction of the maximum allowed latency. One possible way to detect the NTM location is by measuring the temperature fluctuations associated with the magnetic islands using Electron Cyclotron Emission (ECE) radiometry. ECE radiometry is foreseen on ITER, located in equatorial port 9.[8] The spatial dependence of the magnetic field and, consequently, the electron cyclotron frequency, allows for a spatially resolved measurement of the electron temperature.[9] The rotating magnetic island results in a periodic fluctuation of the electron temperature near the NTM rational surface, which can be detected with ECE.[10]

The measured locations, in terms of ECE frequency, require additional techniques to determine the launcher positioning required for accurate deposition. Examples are reconstruction of the magnetic equilibrium and ray tracing to determine launcher settings or detection of the deposition position using the same ECE diagnostic as used for NTM detection.[11][12][13] In this paper, we do not consider measuring ECE along the same line-of-sight of ECCD deposition.[14] A systematic analysis of simulation results is used to determine whether detection of NTMs using ECE is possible for NTM control on ITER.

In this paper, codes are interfaced to analyze NTM detection using the ECE radiometer in the equatorial port plug for a burning H-mode ITER scenario 2 plasma. Simulated ECE and Mirnov coil signals are processed by two detection algorithms, which determine the location of the mode in terms of the ECE frequency. This analysis addresses ECE radiometer settings required for adequate localization, the expected detection time and compare both algorithms.

The next section discusses how simulations of ECE for NTM suppression on ITER are set up and used for simulating the detection of the island location with the algorithms by Berrino et al and Reich et al.[15][16] This section also shows how a realistic temperature perturbation is modelled based on the perturbation of the helical flux. The capabilities of ECE radiometry via the equatorial port are assessed in section 3 , by first deriving a suitable ECE channel spacing to attain the required radial accuracy and secondly determine the detection latency for this spacing. Limits of the simulations, possible improvements of the detection algorithms and possible extensions of the simulations are part of the discussion in section 4 . The final section presents the conclusions.

\section{Simulation set-up}

An overview of the simulation model is given in the first subsection. Most parts of the simulation model are known from literature and described in the appendices. The temperature perturbation resulting from the magnetic islands is discussed in more detail in the second subsection. 


\subsection{Model overview}

The signals detected by an ECE radiometer measuring an ITER plasma are simulated using a combination of models from literature. The simulated signals serve as input for the detection algorithm by Berrino et al.[15] The detection algorithm by Reich et al also relies on Mirnov coil signals.[16] By a suitable combination of Mirnov coil signals, a signal can be created that is sensitive to magnetic perturbations with a specific mode number. Such a signal is simulated using the magnetic perturbation signal $B_{o r}(t) \propto w^{2}(t) \omega(t) \cos (\xi(t))$, with $\xi(t)=\int_{0}^{t} n \omega(\tau) \mathrm{d} \tau$, where the notation used by Reich et al was adopted. Gaussian white noise with $10 \%$ of the Mirnov amplitude $w^{2}(t) \omega(t)$ is added. The relative noise level of $10 \%$ is expected for detection of low order $(m, n)$ MHD modes in ITER.[17] The algorithms detect the island location in terms of a frequency $f_{d e t}$, which can be compared with the actual island location frequency $f_{i s l}$, found from noise-free simulations. Note that $\omega$ describes the rotation of the NTM with respect to the wall. In contrast, the actual island location frequency $f_{i s l}$ indicates the radial location of the island as a result of the spatially dependent EC frequency. Figure 1 shows the components of the model and their interrelations. The components of the model can be replaced by other codes, if those are deemed more fit for the simulation.

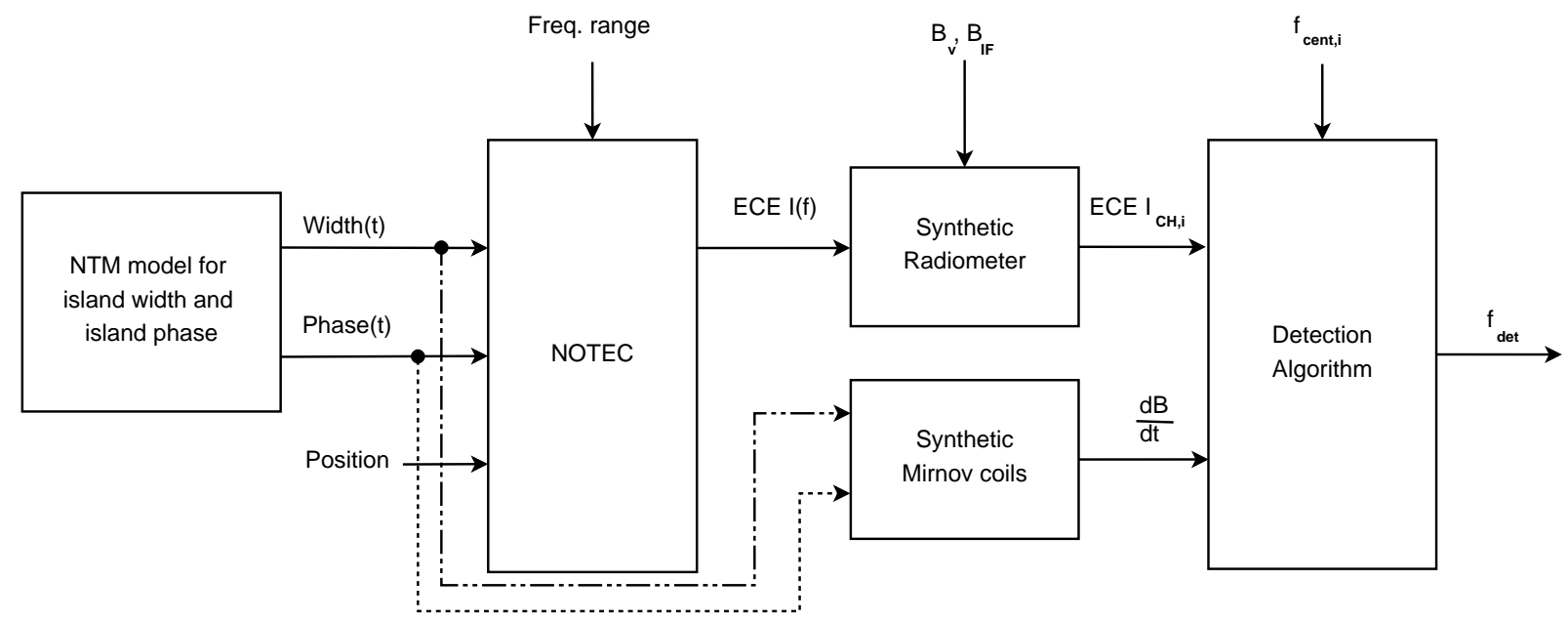

Figure 1: Overview of the components used to model island detection. An NTM model provides island width and phase, as a function of time, and serves, together with the radial mode location, as input for NOTEC. NOTEC calculates the ECE intensity $I(f)$ for a given frequency range. Based on the video bandwidth $B_{v}$ and intermediate frequency bandwidth $B_{I F}$, a synthetic radiometer is simulated, which provides the intensities $I_{C H, i}$ for channels $i$. Mirnov coils are simulated based on the island width and rotation phase. The detection algorithm uses the radiometer, Mirnov coil signals and the centre frequencies of the channels $f_{\text {cent }, i}$, derived from $B_{v}$ and $B_{I F}$, to detect the island location $f_{\text {det }}$.

The evolution of island width and phase is described by an NTM model by Van den Brand et al, which combines island growth described by the Generalized Rutherford Equation and a model for island rotation as put forward by La Haye et 
al.[2][6][18] The island location and temporal evolution of the island width and phase serve as input to the NOn-Thermal ECE Code (NOTEC).[19] This is a code that can calculate both thermal and non-thermal ECE. In this paper, however, we only consider the thermal component of the radiation as measured by the ITER ECE radiometer in the equatorial port plug. Details about NOTEC and the simulation of the ITER burning H-mode scenario 2 plasma is given in Appendix A. The effect of the magnetic islands on the temperature profile is addressed in the following subsection.

NOTEC is used to generate ECE spectra for a set of island widths and phases on a specified frequency range with steps of $50 \mathrm{MHz}$ between individual points in the spectrum. These simulations of fixed island width and phase are combined with the island evolution model to simulate ECE intensity as a function of frequency and time. The noise-free temperature oscillation due to a magnetic island is found by subtracting the average temperature over all island phases from a simulation with a specific phase. The amplitudes of the temperature fluctuations associated with a $3 \mathrm{~cm}$ and $6 \mathrm{~cm}$ island are shown in figure 2 . The radiation at the ECE frequency $f_{i s l}$, with a minimum amplitude of the noise-free temperature oscillation, originates from the region around the island O-point and corresponds to the actual island location. For an optimal suppression of the mode, in case of a radially asymmetric island as modelled here, the ECCD must be deposited on the radius of the island O-point rather than the resonant radius $r_{s}$ of the unperturbed equilibrium.[20] Thermal noise is added to the noise-free ECE, with an amplitude determined by the video bandwidth $B_{v}$ and the intermediate frequency bandwidth $B_{I F}$ of the simulated multichannel radiometer used for detection.[21] The video filter, with bandwidth $B_{v}$, limits the highest amplitude oscillation frequency available in the ECE signals and is approximated by sampling at a fundamental step size $d t=1 /\left(2 B_{v}\right)$. The intermediate frequency bandwidth $B_{I F}$ determines the ECE frequency range measured in a single channel. The number of channels is adjusted to match the simulated frequency range of ECE. The noise levels obtained with $B_{I F}=200 \mathrm{MHz}$ and $B_{I F}=400 \mathrm{MHz}$ are included in figure 2 for a video bandwidth $B_{v}=2 \mathrm{kHz}$. Processing the NOTEC simulations into synthetic radiometer data is addressed in more detail in Appendix $\mathrm{B}$.

The location of the magnetic island in the ECE spectrum is detected using the anti-phase in temperature oscillations at opposite sides of the NTM rational surface (see for instance [15], [22] and [16]) and the minimum in temperature fluctuations itself (see for instance [23]). The algorithms proposed by Berrino et al and Reich et $a l$ are tested on the ITER ECE simulations in the remainder of this paper. These detection algorithms and their implementations are described in Appendix C. With the settings as chosen in Appendix $\mathrm{C}$ and a video bandwidth of $2 \mathrm{kHz}$, the longest averaging time in the algorithms are $100 \mathrm{~ms}$ and $600 \mathrm{~ms}$ for the Berrino and Reich algorithm, respectively.

\subsection{Implementation of magnetic islands}

When a magnetic island is present, the temperature and density profiles no longer depend only on the flux coordinate, but on all three coordinates $\left(r_{c}, \theta, \phi\right)$, where $r_{c}$ is the plasma minor radius in cylindrical coordinates, $\theta$ the poloidal angle and $\phi$ the toroidal angle. The cylindrical radius $r_{c}=\sqrt{\frac{S\left(\psi_{0}, \sigma\right)}{\pi}}$ is determined by the surface $S$ enclosed by the equilibrium helical flux $\psi_{0}$, where $\sigma$ indicates the position relative to the rational flux surface with $\sigma=-1$ for $r_{c}<r_{c, s}$ and $\sigma=+1$ for $r_{c}>r_{c, s}$, where $r_{c, s}$ is the minor radius in cylindrical coordinates of the NTM rational surface. The 


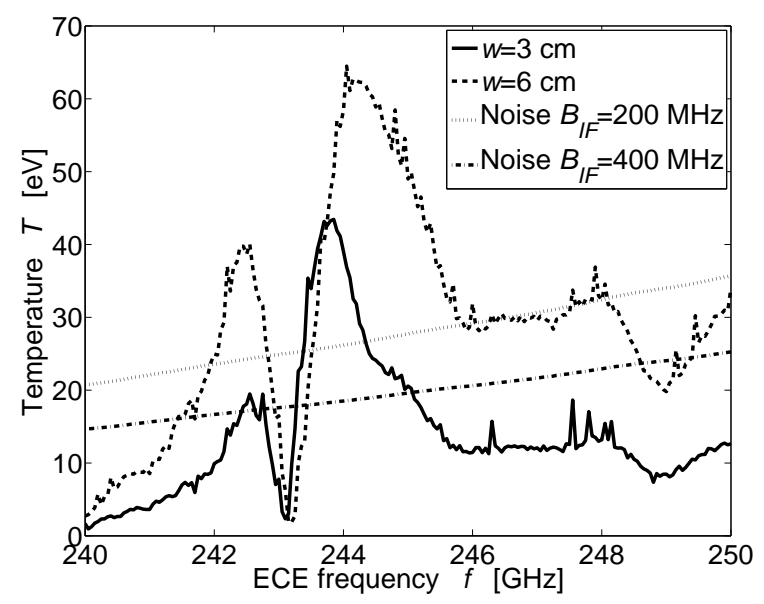

Figure 2: The noise-free temperature oscillation amplitude as a result of a 2/1 magnetic island ( $3 \mathrm{~cm}$ solid, $6 \mathrm{~cm}$ dashed) and the noise level for $B_{v}=2 \mathrm{kHz}\left(B_{I F}=200 \mathrm{MHz}\right.$ dotted, $B_{I F}=400 \mathrm{MHz}$ dashed-dotted) for the second harmonic X-mode range 240-250 GHz. The spikes on the noise-free temperature oscillation result from the relative accuracy of $0.1 \%$ imposed on the simulated absorption coefficient. The spikes are negligible in comparison with the temperature fluctuations.

equilibrium helical flux is defined in such a way that it has a minimum at $r_{c, s}$. The helical flux perturbation by a magnetic island is approximated by Yang et al by[24]

$$
\widetilde{\psi}=\widetilde{\psi}\left(r_{c, s}\right) \frac{r_{c}^{2}}{r_{c, s}^{2}} \frac{\left(1-\frac{r_{c}}{a}\right)^{2}}{\left(1-\frac{r_{c, s}}{a}\right)^{2}} \cos \left(m \theta_{s}+n \phi+\xi_{0}\right),
$$

with $\xi_{0}$ the phase of the NTM and $\theta_{s}$ the straight field line poloidal angle. Using the perturbation amplitude at the rational surface $\widetilde{\psi}\left(r_{c, s}\right)$, the extent of the mode is set to correspond with an island of width $w$, determined as an average of the island width on the low and high field side in the equatorial plane. This form of the helical flux perturbation reproduces the asymmetries in both island extent and the temperature perturbation between $r_{c}>r_{c, s}$ and $r_{c}<r_{c, s}$, as observed in experiments and expected from theory.[25][26][27][20] Previous analyses of the effect of NTMs on the temperature profile have neglected these radial asymmetries in the temperature perturbation.[13][10] [28] Due to the asymmetry, the perturbation is closer to the noise level for $r<r_{s}$ and detection could prove more difficult compared with symmetric perturbations.

Density and temperature are assumed constant on the magnetic flux surfaces described by the perturbed helical flux $\psi=\psi_{0}+\widetilde{\psi}$ and $\sigma$, which holds if the gradients are small and the perpendicular transport is much slower than parallel transport along the flux surface. Due to the small effect of the NTM on the local magnetic field and the small density gradient in ITER, only the perturbation of the temperature has a significant effect on ECE.[29] The radial transport outside the island is assumed to be unaffected, thus maintaining the equilibrium temperature gradients and thereby relating the temperature at $r_{c}>r_{c, s}$ outside the island to the equilibrium temperature. The temperature inside the island is assumed constant due to fast parallel transport 
and equal to the temperature at the outer separatrix, marking the island boundary at $r_{c}>r_{c, s}$. At $r_{c}<r_{c, s}$ outside the island, the temperature drops due to the island by an amount $\Delta T$ with respect to the unperturbed temperature equilibrium, where $\Delta T$ is the temperature difference between the flux surfaces corresponding to the inner and outer separatrix in the unperturbed temperature profile. The temperature $T\left(r_{c}, \theta, \phi\right)$ at position $\left(r_{c}, \theta, \phi\right)$ is therefore related to the equilibrium temperature profile $T_{0}\left(r_{c}\right)$ by

$$
\begin{array}{cl}
\psi\left(r_{c}>r_{c, s}, \theta, \phi\right)>\psi_{\text {sep }} \text { and } \sigma=+1 & T\left(r_{c}, \theta, \phi\right)=T_{0}\left(\sqrt{\frac{S\left(\psi\left(r_{c}, \theta, \phi\right), \sigma\right)}{\pi}}\right) \\
\psi\left(r_{c}, \theta, \phi\right)<\psi_{\text {sep }} & T_{i s l}=T_{0}\left(\sqrt{\frac{S\left(\psi_{\text {sep },+1)}\right.}{\pi}}\right) \\
\psi\left(r_{c}<r_{c, s}, \theta, \phi\right)>\psi_{\text {sep }} \text { and } \sigma=-1 & T\left(r_{c}, \theta, \phi\right)=T_{0}\left(\sqrt{\frac{S\left(\psi\left(r_{c}, \theta, \phi\right), \sigma\right)}{\pi}}\right)-\Delta T \\
& \Delta T=T_{0}\left(\sqrt{\frac{S\left(\psi_{\text {sep }},-1\right)}{\pi}}\right)-T_{0}\left(\sqrt{\frac{S\left(\psi_{\text {sep },+1)}\right.}{\pi}}\right)
\end{array}
$$

with $\psi_{\text {sep }}$ the helical flux at the island separatrices and $S(\psi, \sigma)$ the enclosed area in the poloidal cross section by the flux surface defined by $\psi$ and $\sigma$. Note that in contrast to the assumed constant temperature inside the island, measurements and theory show that the internal temperature profile is not completely flat.[10][25] The best model for temperature fluctuations in magnetic islands in ITER remains to be determined based on local transport coefficients and the structure of magnetic perturbations of the field structure.

\section{Equatorial ECE}

In this section, the detection of the island location, using ECE radiometers in the equatorial plane, is considered. In ITER, the equatorial ECE antenna is located at major radius $R=8.5 \mathrm{~m}$ and height $Z=0.76 \mathrm{~m}$.[8] A value of $6 \mathrm{~cm}$ FWHM is used for both beam widths (see Appendix A for details) which provides an upper estimate to the expected beam width and ensures that beam divergence is negligible. [30] The second harmonic X-mode is used, because it provides higher spatial accuracy than the first harmonic O-mode for equal noise levels.

Note that for a control loop for positioning based on equatorial ECE, methods that determine the deposition position set point, as well as the mirror actuator realizing this set point, should introduce no or small errors in order to achieve the required accuracy. Furthermore, the relativistic shift alone has a larger influence (1.1 GHz on average) than the required accuracy (200 MHz for $2 / 1 \mathrm{NTM}$, see section 3.1) and should therefore be compensated by such methods. Delays introduced by reconstruction and ray tracing codes should also be considered. Reconstruction of the equilibrium and ray tracing or a detection of the deposition position have both been considered.[11][13] Reich et al show the closed loop capabilities of using real-time ray tracing and equilibrium reconstruction for NTM control at ASDEX Upgrade.[12] Although the viability of a reconstruction and ray tracing scheme, in terms of latency, has been shown, realtime reconstruction with an accuracy of a few $\mathrm{mm}$, required for full NTM suppression on ITER, has not yet been demonstrated. These additional steps, although essential in a complete feedback loop, will therefore not be considered here. However, the detection algorithms themselves, relying either on ECE or on a combination of ECE and Mirnov coil data, are evaluated. 
First an appropriate ECE spacing is derived for both $2 / 1$ and $3 / 2$ magnetic islands, based on the required positioning accuracy evaluated for a constant island width of $3 \mathrm{~cm}$. This spacing will be a trade-off between the required radial accuracy (leading to small channel spacing and intermediate frequency bandwidths) and low noise on the detection (typically achieved by having a larger intermediate frequency bandwidth). The spacing is used to generate synthetic ECE data, to which the detection algorithm is employed to determine the detection latency. An example of the simulated signals is given in the second subsection. In the final subsection, the detection latency of both detection algorithms is evaluated for all seed island widths.

\subsection{ECE spacing}

A video bandwidth $B_{v}=2 \mathrm{kHz}$ is used, which suffices for the detection of $3 / 2$ and $2 / 1$ rotating islands, based on the equilibrium toroidal plasma rotation frequency $\omega_{0}$ by La Haye et al $\left(420 \mathrm{~Hz}\right.$ for $2 / 1$ and $578 \mathrm{~Hz}$ for 3/2).[5] Larger values of $B_{v}$ have been used in other ECE designs for ITER.[28]

An accuracy in the ECCD deposition position $\Delta r$ of $7 \mathrm{~mm}$ for $2 / 1$ and $5 \mathrm{~mm}$ for $3 / 2$ magnetic islands should be achieved to ensure full NTM suppression is possible. [6] Based on the ECE frequency $f(R)$ emitted from major radius $R$ along the equatorial ECE sightline, the difference $\Delta f=|f(R)-f(R \pm \Delta r)|$ is evaluated to arrive at the required frequency accuracy for a given required spatial accuracy $\Delta r$. This results in an accuracy requirement of $200 \mathrm{MHz}$ and $150 \mathrm{MHz}$ for the detected ECE frequency of the $2 / 1$ and $3 / 2$ magnetic islands, respectively.

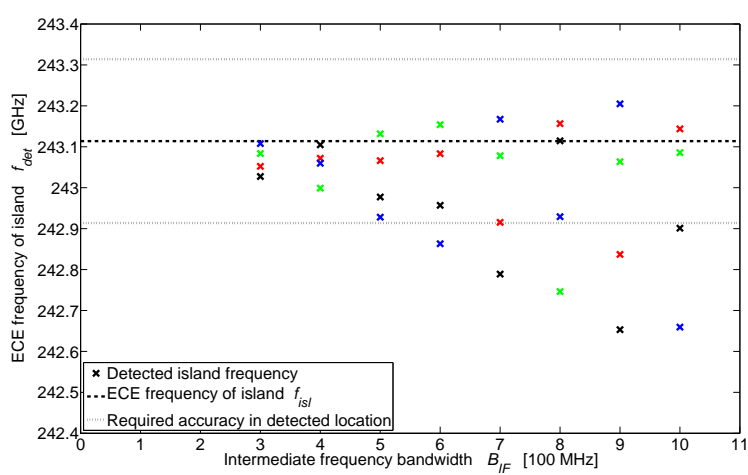

(a) Berrino

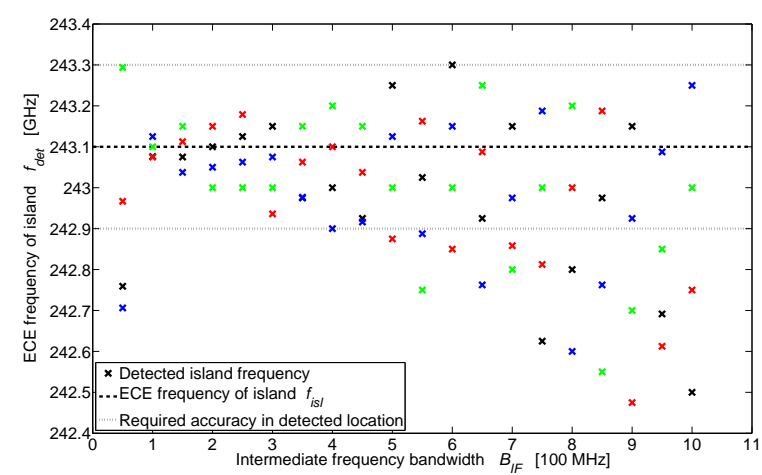

(b) Reich

Figure 3: The detected ECE island frequency as a function of $B_{I F}$ for a magnetic $m / n=2 / 1$ island of $3 \mathrm{~cm}$ rotating at $95.5 \mathrm{~Hz}$, for the algorithms by Berrino et al (left) and Reich et al (right). The centre frequency of the first band is located at $240 \mathrm{GHz}+(c+0.5) B_{I F}$, where $c$ is an offset value of 0,1/4, 2/4 or 3/4 (shown as black, red, green and blue crosses, respectively). The actual island frequency and the accuracy bounds are indicated by dashed and dotted lines respectively.

Figure 3 shows the detected ECE frequency, for a $3 \mathrm{~cm} m / n=2 / 1$ island rotating at $95.5 \mathrm{~Hz}$, as a function of intermediate bandwidth $B_{I F}$ for both detection algorithms. 
The channel spacing is assumed equal to $B_{I F}$ and ECE is collected in a range from 240$250 \mathrm{GHz}$. The centre frequency of the first channel is located at $240 \mathrm{GHz}+(c+0.5) B_{I F}$, where $c$ is an offset value of $0,1 / 4,2 / 4$ or $3 / 4$, to assess the effect of a different channel spacing related to the island location. The figures show detection within the accuracy limits for a limited number of intermediate frequency bandwidths. For the algorithm by Berrino et al, accurate detection is possible for intermediate frequency bandwidths of $300 \mathrm{MHz}$ and $400 \mathrm{MHz}$. For lower intermediate frequency bandwidths, the NTM is no longer detected. The algorithm by Reich et al shows accurate detection for intermediate frequency bandwidths ranging from $100 \mathrm{MHz}$ to $400 \mathrm{MHz}$. For larger intermediate frequency bandwidths, the location is not detected accurately enough due to the lack of interpolation between radiometer channels. At an intermediate frequency bandwidth of $50 \mathrm{MHz}$ NTMs are detected, but are not accurately localized. The failing detection at low intermediate frequency bandwidths for both algorithms is a result of the increased thermal noise, as illustrated in figure 2 .

The detection algorithm by Berrino et al assumes that the temperature fluctuation is the result of the rotating island. However, because the noise at ECE frequencies below $243 \mathrm{GHz}$ is comparable to the temperature fluctuation of a $3 \mathrm{~cm}$ island, an offset towards lower ECE frequencies is observed in figure 3 . The temperature fluctuation for larger islands is well above the noise level.

For the $3 / 2 \mathrm{NTM}$, the dependencies of the detected island frequency on the intermediate frequency bandwidth are similar. In this case, ECE is simulated from $240 \mathrm{GHz}$ to $260 \mathrm{GHz}$ to include the NTM which has an island frequency of $248.76 \mathrm{GHz}$. A spacing of $B_{I F}=400 \mathrm{MHz}$ also provides satisfactory results for $3 / 2$ magnetic island detection for the algorithm by Berrino et al. For the algorithm by Reich et al an intermediate frequency of $300 \mathrm{MHz}$ is required to ensure $3 / 2$ NTMs are also detected accurately. The performance of the algorithm by Reich et al is likely to improve by applying an interpolation as done in the algorithm by Berrino et al.

\subsection{Island detection example}

NOTEC simulations of ECE spectra as a function of island width and phase are combined with a temporal evolution of island width and island rotation, following the model by Van den Brand et al. [6] Figure 4 shows the evolution of island width, island rotation, ECE signals and the detected island location. The channel spacing for the algorithm by Berrino et al, with an intermediate frequency bandwidth of $400 \mathrm{MHz}$ and channel centre frequencies ranging from $240.2 \mathrm{GHz}$ to $249.8 \mathrm{GHz}$, is shown in the figure below. The video bandwidth is $2 \mathrm{kHz}$. Only a limited part of the channels is shown. A channel spacing with an intermediate frequency bandwidth of $300 \mathrm{MHz}$ is used for the algorithm by Reich et al.

An island of $2 \mathrm{~cm}$ is seeded at $t=1 \mathrm{~s}$. Island growth is simulated until the island locks. As the island grows the average temperature in the plasma core decreases and the temperature fluctuations increase in amplitude. Outside the island, the temperature fluctuations are larger near the island than further away and at radii smaller than $r_{s}$ the temperature fluctuations are larger compared to the fluctuations at $r>r_{s}$. This is a result of the imposed asymmetry on the perturbed helical flux and is consistent with experimental observations.

For 2/1 magnetic islands, the island location $f_{i s l}$, indicated by a dashed black line in the bottom plot, shifts to higher frequencies as the island width increases. This is consistent with the radial shift of the island O-point as a consequence of the asymmetry 


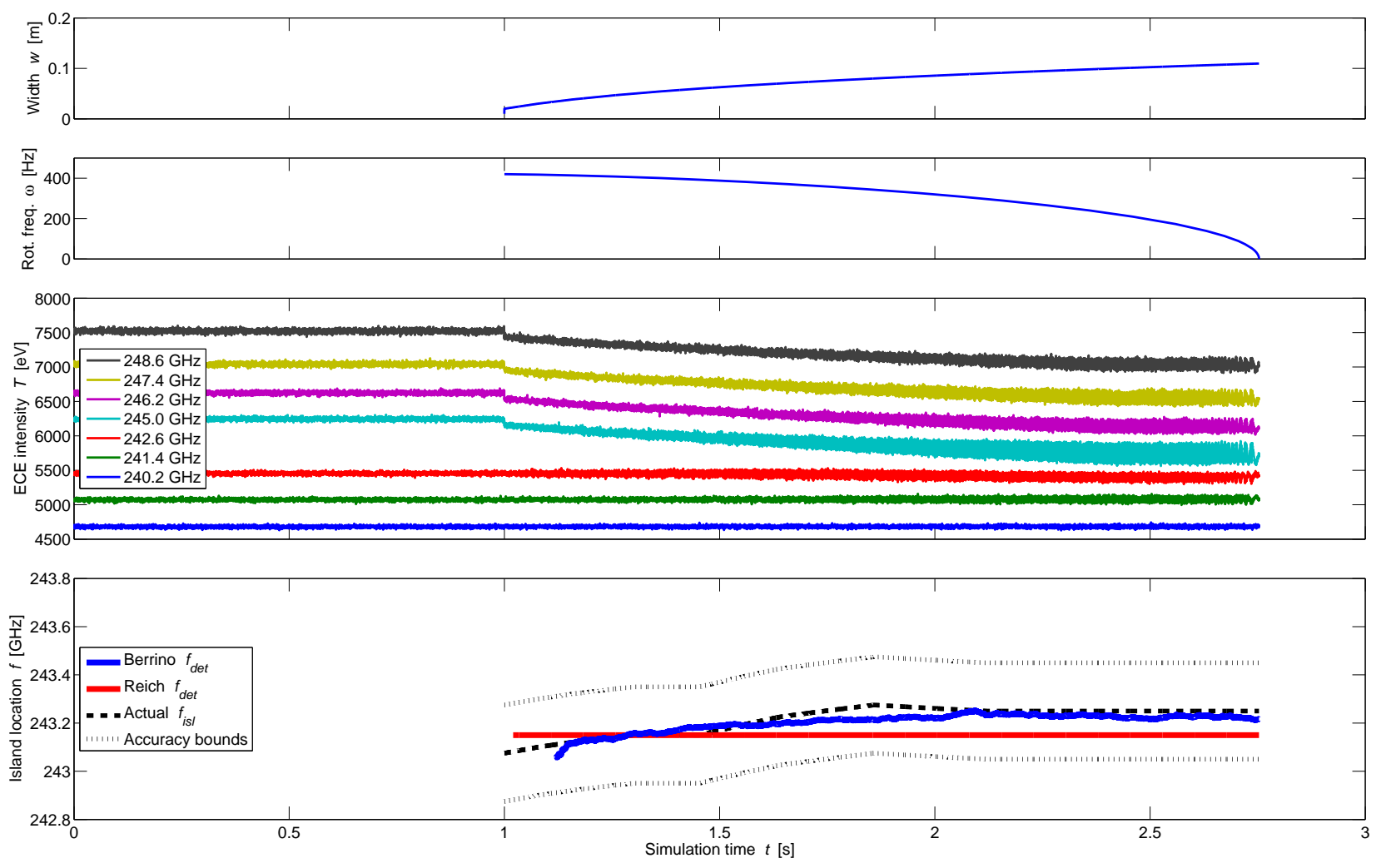

Figure 4: 2/1 NTM seeded with $2 \mathrm{~cm}$ at $1 \mathrm{~s}$, growing in accordance with the polarization model with $w_{\text {marg }}=2 \mathrm{~cm}$. The simulation is stopped when the mode locks. From top to bottom, the boxes show island width, toroidal rotation frequency, simulated ECE signals and the actual and detected island locations by both algorithms. 7 of the 25 simulated ECE channels for the algorithm by Berrino et al are shown.

in the perturbed flux across $r_{s}$. This difference amounts to $200 \mathrm{MHz}$, going from 2 to $9 \mathrm{~cm}$ island widths, and needs to be compensated, because it is on the order of the required accuracy. The accuracy of $f_{i s l}$ is limited due to the limited frequency step size of $50 \mathrm{MHz}$ in the underlying ECE spectrum simulations. The dotted lines show the upper and lower bound on the detected island location to maintain an estimate within $200 \mathrm{MHz}$ and hence to ensure the required deposition accuracy is reachable. The ECE frequency $f_{\text {det }}$ is determined using the algorithms presented in Appendix C. The island is detected within $111 \mathrm{~ms}$ by the algorithm by Berrino et al and the detected location remains well within the accuracy bounds. The algorithm by Reich et al responds almost instantly to the Mirnov signals as soon as the island is seeded. It takes the algorithm $20 \mathrm{~ms}$ to accumulate sufficient data for an accurate detection of the location, which remains accurate in the remainder of the island evolution. 


\subsection{Detection latency with equatorial ECE}

In section 3.1, a video bandwidth $B_{v}$ of $2 \mathrm{kHz}$ and intermediate frequency bandwidths $B_{I F}$ of $400 \mathrm{MHz}$ and $300 \mathrm{MHz}$, for the algorithms by Berrino et al and Reich et al respectively, are shown to be required for accurate detection of $2 / 1$ and $3 / 2$ magnetic islands. Using a channel layout, with these settings and the offset value $c$, mentioned in the first subsection, set to zero, the detection latency is determined. ECE is simulated from $240 \mathrm{GHz}$ to $250 \mathrm{GHz}$ for the $2 / 1 \mathrm{NTM}$ and to $260 \mathrm{GHz}$ for the $3 / 2 \mathrm{NTM}$. The detection latency is defined as the time difference between island seeding and the time at which the detected island location is within the required accuracy of $200 \mathrm{MHz}$ or $150 \mathrm{MHz}$ for the 2/1 and 3/2 NTM, respectively, and remains accurate for the remainder of the simulation run. The detection latency is determined for seed island widths varied from $2 \mathrm{~cm}$ up to $11 \mathrm{~cm}$ for $2 / 1$ NTMs and up to $12 \mathrm{~cm}$ for $3 / 2$ NTMs. Each simulation is repeated 15 times with different noise realizations. The mean, minimum and maximum detection latencies, determined from the 15 noise realizations, for both detection algorithms as a function of seed island width are shown in the bottom plots of figure 5 and figure 6 for the $2 / 1$ and $3 / 2$ NTM respectively. In both plots, the detected latencies are accompanied by the maximum allowed latency as determined by Van den Brand et al. [6]

Figures 5 shows that for the 2/1 NTM the algorithm by Reich et al shows little dependence on the seed island width. Conversely, for the $3 / 2$ NTM, the detection latency depicted in figure 6 shows a slightly larger detection latency for both small and large seed islands. The algorithm by Berrino et al shows an increasing detection latency for both $2 / 1$ and $3 / 2 \mathrm{NTMs}$ due to the reduced performance of this algorithm for larger island widths. For small islands, the fluctuation amplitude is low, resulting in an increased detection latency for both algorithms. A special case is a seed island width of $2 \mathrm{~cm}$, for which the mode needs to grow to become detectable. Both detection algorithms show a detection latency below $250 \mathrm{~ms}$ for all simulated noise realizations.

Detection within the maximum allowed latency is possible for islands up to $9 \mathrm{~cm}$ and $11 \mathrm{~cm}$ for the $2 / 1$ and $3 / 2$ NTM respectively. For the smaller seed island widths, the detection latency varies only slightly and is. for the largest part of the range, only a smaller factor of the maximum allowed latency. If only detection latency is taken into account, the largest seed island width that can be suppressed is reduced with only $1 \mathrm{~cm}$ compared to a situation where all latencies are zero. The algorithm by Reich et al shows the shortest detection latencies, but also incorporates more information about the plasma using the Mirnov coil signals. Tests with the algorithm by Berrino et al showed that a variation in the offset $c$ results in an increased average detection latency of $250 \mathrm{~ms}$ and $400 \mathrm{~ms}$ for the $2 / 1$ and $3 / 2 \mathrm{NTM}$, respectively. If $B_{I F}$ where to be increased, the radiometer noise would decrease. Assuming that $B_{I F}$ is not so large that the perturbations peaks on opposite sides of the island are in a single channel, this would result in a smaller detection latency. However, for larger $B_{I F}$ the detection is not able to achieve the accuracy required for full suppression.

\section{Discussion}

The simulations presented in this paper include what are expected to be the relevant dynamics for NTM detection using ECE. The physics that was excluded from the simulations is discussed in this section. The limitations of the island location sensor are also discussed, focussing on possible improvements of the algorithm. The last 

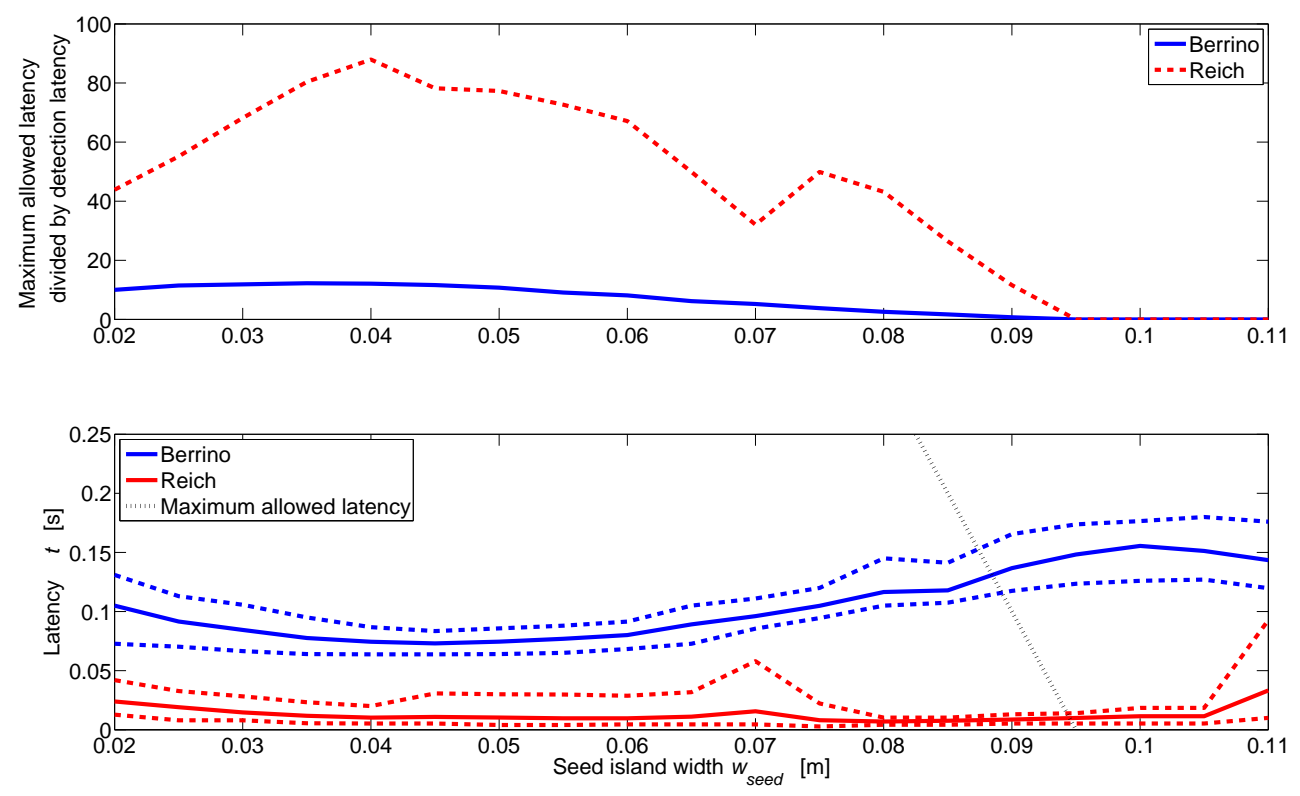

Figure 5: Detection latency for 2/1 NTMs, as function of the seed island width, for island location using equatorial ECE with detection algorithms by Berrino et al and Reich et al. The top figures show the maximum allowed latency, derived by Van den Brand et al[6], divided by the average detection latency over 15 runs with different noise realizations for both the algorithm by Berrino et al (solid) and Reich et al (dashed). The bottom figures show the mean detection latency (solid) and the shortest and longest detection latency (dashed) determined from 15 runs with different noise realizations for both the algorithm by Berrino et al (blue) and Reich et al (red). For comparison, the maximum allowed latency is depicted as a dotted line.[6]

paragraph is dedicated to the extension of simulations to encompass an entire ITER NTM control loop, to fully assess latency, accuracy and compatibility of the different components.

The used ECE channel layout is idealized, assuming that there is no overlap in ECE frequencies detected with adjacent channels. The video filter is approximated by sampling at time intervals $1 /\left(2 B_{v}\right)$ to ensure frequencies up to $B_{v}$ are present in the measured ECE signals. In practice, radiometers show a lower gain for ECE frequencies and video frequencies near the edge of the band. ECE frequency overlap between neighbouring ECE channels is also observed in practice. For comparison, a sixth-order Chebyschev or Butterworth filter will collect approximately $85 \%$ of its radiation inside the pass band.[31] However, $96 \%$ of the radiation comes from a region of 1.2 times the bandwidth. This could result in a correlation of $11 \%$ between two adjacent channels. As the sophistication of models and the demands on simulation accuracy increases, these overlap effects could be incorporated as well.

The noise on the Mirnov coils is now implemented relative to the Mirnov signal 

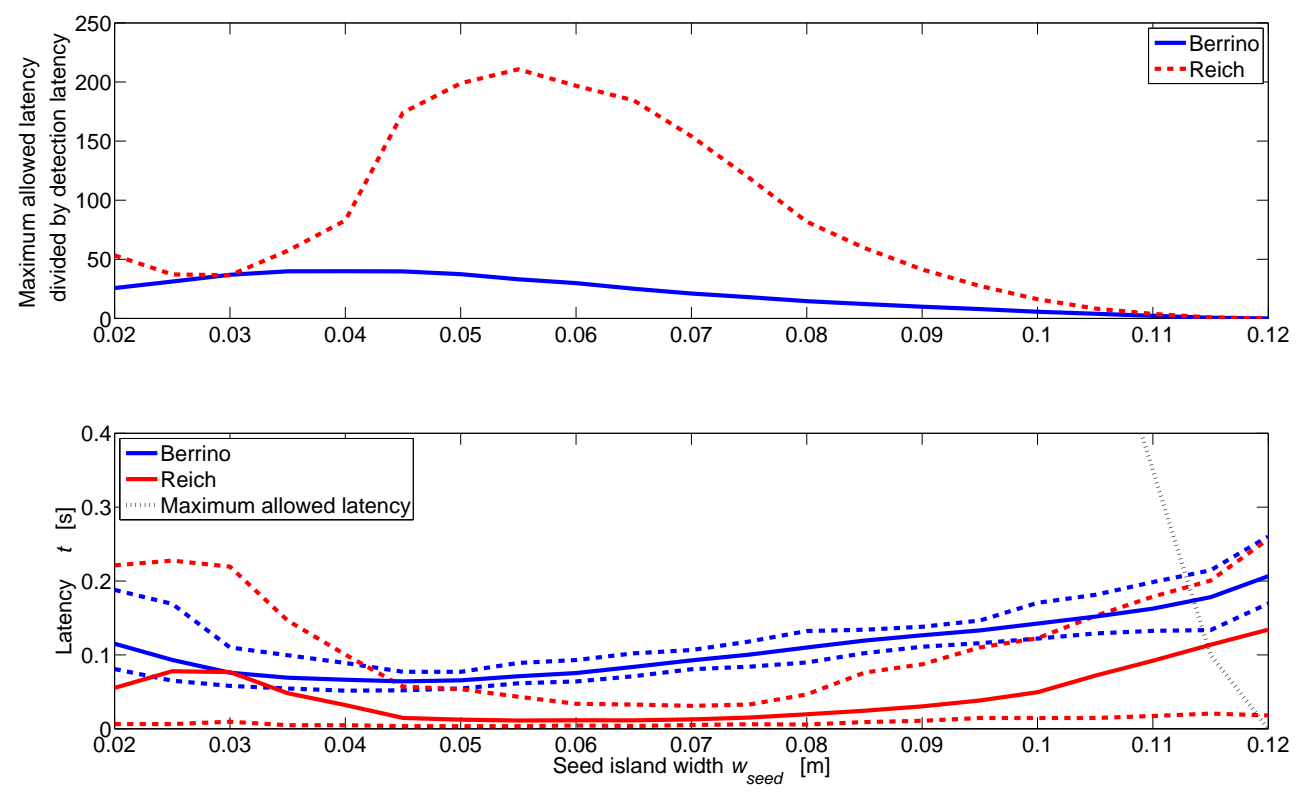

Figure 6: Detection latency for 3/2 NTMs, as function of the seed island width, for island location using equatorial ECE with detection algorithms by Berrino et al and Reich et al. The top figures show the maximum allowed latency, derived by Van den Brand et al[6], divided by the average detection latency over 15 runs with different noise realizations for both the algorithm by Berrino et al (solid) and Reich et al (dashed). The bottom figures show the mean detection latency (solid) and the shortest and longest detection latency (dashed) determined from 15 runs with different noise realizations for both the algorithm by Berrino et al (blue) and Reich et al (red). For comparison, the maximum allowed latency is depicted as a dotted line.[6]

amplitude. The actual noise level, which is expected to be constant, is not yet known and its determination might prove difficult without actual ITER measurements. As a result of the relative noise level, no noise was present in the absence of the island. Adding noise without the presence of the island resulted in false detection with the algorithm by Reich et al. However, these false detections occur infrequently and with varying detected locations and could therefore be filtered out easily. The effect of temperature fluctuations inside the island needs to be assessed as well.

Only the thermal noise by the uncorrelated ECE and noise on the Mirnov coils have been taking into account. Other noise sources such as edge density fluctuations and turbulent temperature fluctuations or transients such as sawteeth or ELMs are not incorporated.[32][33] The presence of multiple NTMs could also disturb the detection of the location. The algorithm by Reich et al could probably handle multiple modes better, because only the magnetic perturbations by a single NTM, obtained by a proper combination of multiple Mirnov coil signals, are used. The effect of these additional noise sources and transients on NTM detection can be studied by examining how they 
influence EC wave propagation and inferring the effect on ECE in particular. At this moment no conclusions can be drawn regarding the influence of other noise sources on island detection using ECE on ITER. However, it should be noted that the algorithm by Reich et al has been tested in the experimental environment of ASDEX Upgrade in which among other perturbations sawteeth and ELMs are present.[12]

The radial accuracy that can be reached is limited by the thermal noise for both detection algorithms. The thermal noise increases with decreasing intermediate frequency bandwidth. The detection algorithm by Berrino et al showed increasing latencies for larger seed island widths. The larger islands reduce the fluctuations due to temperature equilibration in a larger frequency range, thereby decreasing the correlation signals between the channels measuring radiation coming from inside the island. Both issues can be tackled by a multi-scale detection method.[34] By using larger intermediate frequency bandwidths, the signal-to-noise ratio improves and small islands can be detected earlier. Larger intermediate frequency bandwidths also imply a larger channel spacing, thereby collecting less of the equilibrated temperature inside the island relative to the ECE detected outside the island. A drawback is the reduced spatial accuracy in the detected location, which would still require the lower intermediate frequency bandwidth and hence incorporation of multiple intermediate frequency bandwidth scales in the algorithm. A direct digitized radiometer system with a real-time tunable intermediate frequency bandwidth, as designed by Bongers et al, could be beneficial for multi-scale detection. [35] A similar result can be achieved with a conventional radiometer with a small intermediate frequency bandwidth of which the detected ECE of multiple channels is added together to obtain ECE detected with a larger intermediate frequency bandwidth.

The simulations presented in this paper address only two components of a control loop for NTMs: the plasma with the island and the detector. The design of a controller is a much more challenging task, which requires the modelling of the full control loop. In such a control loop, the detected island location $f_{\text {det }}$ must first be translated into a requested angle for the ECCD steering mirror, required to deposit the power at the proper flux surface. Next, the dynamics of the steering mirror as identified for a prototype by Collazos et al[36], which serves as the main actuator, must be incorporated in the control loop. Moreover, such simulations should also incorporate the deposited ECCD power in a consistent way in the evolution of both the detected ECE spectrum and the amplitude and phase of the NTM. Note that this proposed control loop addresses only the control of NTMs before locking occurs. Modelling of additional actuators for locked mode control and determination of the detection latency for such a control loop is required, if locked modes are considered a problem for ITER operations.

The detected island location $f_{\text {det }}$ can be translated to a requested angle for the ECCD steering mirror in two ways. One way is using a combination of equilibrium reconstruction and ray tracing. Plasma measurements are combined to determine the magnetic, temperature and density profiles. Using this realtime determined equilibrium the exact position of the NTM can be determined. Using realtime ray tracing, the mirror settings required to deposit ECCD on the NTM position is calculated. A control loop based on equilibrium reconstruction and real time ray tracing is implemented and tested by Reich et al for the ASDEX Upgrade tokamak in Germany.[12]

The second way is to measure both the location of the NTM and the location of the deposited power with the same ECE diagnostic. Detection of modulated ECCD 
with the same ECE diagnostic as the one used for magnetic island island detection is described by Manini et al.[13] Alternatively, ECE can be detected along the same line-of-sight as the ECCD deposition, as was first proposed by Westerhof et al.[14] This has the advantage that only the island location needs to be detected. Hennen et al showed realtime NTM control on TEXTOR with same line-of-sight ECE.[22] An ITER compatible same line of sight ECE diagnostic has been presented by Bongers et al. [37]

\section{Summary and conclusions}

Simulation codes are combined to model mode detection using ECE for NTM control. ECE coming from an ITER plasma containing a magnetic island was simulated and fed to detection algorithms. The perturbed temperature profile not only takes into account the flattening of the temperature inside the island, but also the perturbation of the flux surfaces outside the island. The flux surface perturbation as well as the temperature perturbation itself are radially asymmetric around the rational flux surface, thereby providing a realistic temperature perturbation that is consistent with observations in present-day experiments.[25][26][27] Simulated ECE spectra, measured at frequencies $50 \mathrm{MHz}$ apart, are combined in ECE channels and thermal noise is added based on the intermediate frequency and video bandwidths. A set of ECE spectra with varying island width and phase are combined with models for the evolution of the island width and rotation to arrive at time dependent ECE signals. The actual island location, indicated by the frequency of minimum temperature fluctuation in the noisefree spectra, should be accurately detected for NTM control. The algorithms by Berrino et al and Reich et al are used for island detection.[15][16] The algorithm by Reich et al also used synthetic Mirnov coil data, simulated using the island width and phase.

For ECE in the equatorial port plug, the required ECE channel layout to attain a detection accuracy of $7 \mathrm{~mm}$ for the $2 / 1$ and $5 \mathrm{~mm}$ for the $3 / 2 \mathrm{NTM}$ is investigated. This detection accuracy is equal to the required deposition accuracy for full suppression. [6] A video bandwidth of $2 \mathrm{kHz}$ is used which suffices for the detection of $2 / 1$ and $3 / 2$ NTMs based on a model for mode rotation by La Haye et al.[18] The detection latency is determined for seeded islands, growing in accordance with the polarization model for the saturation of the bootstrap island growth at small island widths.

A sufficient detection accuracy for $3 \mathrm{~cm}$ islands is attained with a $400 \mathrm{MHz}$ and $300 \mathrm{MHz}$ intermediate frequency bandwidth for both the $2 / 1$ and $3 / 2 \mathrm{NTM}$, for the algorithms by Berrino et al and Reich et al respectively. The channel spacing is taken equal to the intermediate frequency bandwidth. The intermediate frequency bandwidth provides a trade-off between radial accuracy (attained with a small bandwidth) and detection latency (achieved with low noise for large bandwidths). For large islands ECE channels are located partially in the island, thereby decreasing the detected temperature oscillations and reducing the performance of the algorithm by Berrino et al. The simulations show that accurate detection of magnetic islands within $250 \mathrm{~ms}$ is possible with both algorithms for islands up to $9 \mathrm{~cm}$ and $11 \mathrm{~cm}$ for the $2 / 1$ NTM and $3 / 2$ NTM respectively.

The algorithm by Reich et al showed faster detection times than the algorithm by Berrino et al. In contrast, the algorithm by Berrino et al uses only ECE information resulting in a longer detection latency. It should be noted that not all relevant noise and perturbation sources, such as other MHD modes, are included and that 
the detection algorithms could both be improved further. Nevertheless, this paper provides a systematic way to compare detection algorithms, based on accuracy and detection latency, and judge whether requirements are met for NTM control on ITER.

\section{Acknowledgments}

The help of Matthias Reich in setting up and evaluating simulations with the algorithm by him and his coauthors is greatly appreciated.

The work in this paper has been performed in the framework of the NWO-RFBR Centre of Excellence on Fusion Physics and Technology (grant 047.018.002). This work, supported by the European Communities under the contract of association between EURATOM-FOM, was carried out within the framework of the European Fusion Program. The views and opinions expressed herein do not necessarily reflect those of the European Commission.

\section{Appendix A. NOTEC[19]}

Based on the magnetic field, density and temperature profiles, NOTEC calculates the ECE collected by an antenna facing the plasma. For thermal ECE, NOTEC solves the radiation transport equation

$$
\frac{d}{d s} I(f)=\frac{\alpha(f) f^{2} k_{B} T}{c^{2}}-I(f) \alpha(f)
$$

along the path of EC wave propagation in a toroidal geometry, where $s$ is the path length, $I$ the ECE intensity, $f$ the ECE frequency, $\alpha$ the absorption coefficient, $k_{B}$ the Boltzmann constant and $T$ the electron temperature.[38][19] The ECE path is traced back, starting at the antenna, to the EC emission region using the RAYS code implementation in NOTEC, providing geometrical optics ray tracing using the local density, temperature and magnetic field.[39] The antenna is specified by three position coordinates $X_{a}, Y_{a}, Z_{a}$, a toroidal $\phi_{a}$ and a poloidal $\theta_{a}$ antenna angle, two transverse beam widths $w_{t}$ and $w_{p}$, associated with the $\phi_{a}$ and $\theta_{a}$ angles respectively, and beam divergence in toroidal $\delta \phi$ and poloidal $\delta \theta$ direction. Up to 21 rays can be used to simulate an antenna pattern, where for every set of four rays a constant weighting is used, determined by the two-dimensional Gaussian beam intensity with widths $w_{t}$ and $w_{p}$. The divergence of individual rays of a beam is given by $\Delta \phi=h / w_{t} \delta \phi$ and $\Delta \theta=l / w_{p} \delta \theta$ with $h$ and $l$ the toroidal and poloidal distance to the antenna centre.

The magnetic field in the poloidal plane is specified using two dimensional splines based on the burning H-mode ITER scenario 2 plasma.[29][40] Density and temperature are specified on the flux coordinates derived from the magnetic field topology. Equation A.1 is solved using the absorption coefficient $\alpha(f)$ determined by iteratively solving the biquadratic equation with a relative accuracy of $0.1 \%$ for the refractive index derived by Bornatici et al. [9] The thermal absorption, equilibrium and ray tracing routines, which form the core of NOTEC, are also part of the ray tracing code TORAY, which has been extensively benchmarked for the ITER scenario 2 plasma.[41][42][40] NOTEC produces the ECE intensity for a set of specified ECE frequencies and also provides the path travelled by individual rays. 


\section{Appendix B. Synthetic ECE measurements}

ECE is commonly detected using multichannel radiometers. A radiometer is simulated based on its video bandwidth $B_{v}$ and the intermediate frequency bandwidth $B_{I F}$. [21] The video filter, with bandwidth $B_{v}$, limits the highest amplitude oscillation frequency available in the ECE signals and can be approximated by sampling at a fundamental step size $d t=1 /\left(2 B_{v}\right)$, assuming frequencies larger than $B_{v}$ are not present in the data. The intermediate frequency bandwidth $B_{I F}$ determines the ECE frequency range that is measured in a single channel. Thermal ECE fluctuations result in noise detected by the radiometer given by

$$
\frac{\Delta T}{<T>}=\sqrt{\frac{2 B_{v}}{B_{I F}}}
$$

where $\langle T\rangle$ is the mean temperature and $\Delta T$ the standard deviation of the Gaussian white temperature noise.

A synthetic measurement is obtained by combining ECE spectra of a magnetic island evolving over time with an ECE channel layout, determined by the radiometer settings $B_{v}, B_{I F}$, channel spacing and the number of channels. In this paper, measurements at frequencies $f_{50}$, separated by frequency intervals of $50 \mathrm{MHz}$, are assumed to represent the ECE signal for a frequency range $f_{50}-25 \mathrm{MHz}<f<$ $f_{50}+25 \mathrm{MHz}$. At time instances $t$, the simulated ECE spectrum is interpolated for the island width $w(t)$ and phase $\xi(t)=\int_{0}^{t} n \omega(\tau) \mathrm{d} \tau$, based on a database of NOTEC simulations for different island widths and phases. Gaussian white noise with an amplitude specified by equation B.1, using $B_{I F}=50 \mathrm{MHz}$, is added to all simulated ECE intensities for the frequencies $f_{50}$. The Gaussian white noise is generated using an internal Matlab Simulink pseudo-random number generator with an initial seed. Every ECE channel has a separate generator with a different seed (making noise across channels independent). To generate a different realization, a new set of seeds is used for all random number generators. The random numbers are generated with a mean of zero and a standard deviation given by equation B.1. The resulting ECE intensities $I_{50}$, including thermal noise, at frequencies $f_{50}$ are added to form the channel intensity

$$
I_{C H}=\int_{f_{\text {cent }}-B_{I F} / 2}^{f_{\text {cent }}+B_{I F} / 2} I_{50}(f) \mathrm{d} f,
$$

where $f_{\text {cent }}$ is the channel centre frequency and $B_{I F}$ the intermediate frequency bandwidth of the simulated channel.

\section{Appendix C. Detection algorithms for island location}

The algorithms by Berrino et al and Reich et al both rely on the difference in the phase of the temperature oscillation on opposite sides of the rational flux surface.[15][16] The algorithms and their implementation for the simulations are described in this appendix.

\section{Algorithm by Berrino et al}

The algorithm by Berrino et al identifies the location of the mode based on the local minimum in the cross correlation between adjacent ECE channels on opposite sides of 
the rational flux surface. When the second derivative of the cross correlation exceeds a preset threshold, the associated minimum in the cross correlation is assumed to be caused by the presence of an island. Its location is then determined by interpolating the zero crossing of the first derivative of the cross correlations. Below we generalize the algorithm of Berrino et al[15] to arbitrary frequency steps between ECE channels.

First a moving average of $N_{\text {mov }}$ measurements is subtracted to arrive at a temperature fluctuation

$$
\delta T(t, j)=T(t, j)-\frac{1}{N_{\text {mov }}} \sum_{i=1}^{N_{\text {mov }}} T(t+(1-i) d t, j)
$$

for all channels $j$, where $T(t, j)$ is the measured electron temperature in channel $j$ at time $t$ and $d t$ the sampling time, limited by the video bandwidth $B_{v}$ as reported in Appendix B. This fluctuation is normalized using the square root of a moving average over $N_{\text {avg }}$ samples of the squared temperature fluctuation resulting in

$$
\delta T_{\text {norm }}(t, j)=\frac{\delta T(t, j)}{\sqrt{\frac{1}{N_{\text {avg }}} \sum_{i=1}^{N_{\text {avg }}} \delta T^{2}(t+(1-i) d t, j)}} .
$$

The correlation between two adjacent ECE channels is calculated as the average over $N_{a c}$ measurements of the product of the two normalized temperature fluctuations using

$$
P(t, j)=\frac{1}{N_{a c}} \sum_{i=1}^{N_{a c}} \delta T_{n o r m}(t+(1-i) d t, j+1) \delta T_{n o r m}(t+(1-i) d t, j),
$$

which are defined at frequencies

$$
f_{P}(j)=\frac{1}{2}\left(f_{\text {cent }}(j+1)+f_{\text {cent }}(j)\right),
$$

for $j=1 \ldots N_{c h}-1$ with $N_{c h}$ the number of channels and $f_{c e n t}(j)$ the centre frequency of channel $j$. The discrete first derivative with respect to the frequency

$$
D_{1}(t, j)=\frac{P(t, j+1)-P(t, j)}{f_{P}(j+1)-f_{P}(j)}
$$

is calculated for $j=1 \ldots N_{c h}-2$ at frequencies

$$
f_{D_{1}}(j)=\frac{1}{2}\left(f_{P}(j+1)+f_{P}(j)\right)
$$

and used to calculate the discrete second derivative

$$
D_{2}(t, j)=\frac{D_{1}(t, j+1)-D_{1}(t, j)}{f_{D_{1}}(j+1)-f_{D_{1}}(j)}
$$

for $j=1 \ldots N_{c h}-3$. When the second difference $D_{2}$ exceeds a threshold $D_{2, t h r}$, the anti-correlation is taken to indicate the presence of an island. The island is taken to be located at the zero crossing of $D_{1}$, which is found through linear interpolation with

$$
f_{\text {det }}(t)=\frac{D_{1}\left(t, j_{\max }\right) f_{D_{1}}\left(j_{\max }+1\right)-D_{1}\left(t, j_{\max }+1\right) f_{D_{1}}\left(j_{\max }\right)}{D_{1}\left(t, j_{\max }\right)-D_{1}\left(t, j_{\max }+1\right)},
$$

where $j_{\max }$ is the index number of the largest $D_{2}$ entry.

The averages are implemented in MathWorks Simulink using digital filters $G(z)=\frac{K}{z-(1-K)}$, where $K=1 /(N+1)$ and $1 / z$ is the unit delay operator.[31] With 
$B_{v}=2 \mathrm{kHz}, N_{\text {mov }}=40$ corresponds to an average over $10 \mathrm{~ms}$ resulting in an average over an entire period for islands with a rotation frequency of $100 \mathrm{~Hz}$ and larger. For the normalization average and the average in the correlation, values of $N_{\text {avg }}=80$ and $N_{a c}=400$ are chosen, where the relationship between $N_{m o v}, N_{a v g}$ and $N_{a c}$ is the same as used by Berrino et al.[15] A proper choice for $D_{2, t h r}$ ensures noise is not wrongly identified as an island. A value of $\frac{0.6}{\left(f_{\text {cent }}(2)-f_{\text {cent }}(1)\right)^{2}}$ is found to yield no false detections.

By setting $N_{m o v}=N_{a v g}$ and a decrease of $N_{a c}$ and $D_{2, t h r}$, the detection latency can be decreased, which is accompanied by a decrease in accuracy of the detected location.

\section{Algorithm by Reich et al}

The algorithm by Reich et al detects the anti-phase at the NTM rational flux surface by multiplying the ECE oscillation with a signal, composed using a combination of Mirnov coil signals, and a $90^{\circ}$ phase-shifted signal.[16] This requires a synthetic signal $B_{\text {or }}(t) \propto$ $w^{2}(t) \omega(t) \cos (\xi(t))$, a phase-shifted signal provided by $B_{p s}(t) \propto w^{2}(t) \omega(t) \sin (\xi(t))$ and the electron temperature $T_{i, e}(t)$ for channels $i$ from 1 to the number of channels $N_{c h}$. Reich et al use a Hilbert transform to obtain $B_{p s}(t)$ from $B_{o r}(t)$. Instead, in this implementation the analytical solution $\sin (\xi(t))$ of the Hilbert transform of $\cos (\xi(t))$ is implemented directly. This could result in a lower detection latency than what would be achieved when the Hilbert transform needs to be calculated on the noisy simulated signal $B_{o r}(t)$.

Using FIR filters, the averages $\overline{B_{o r}(t)}, \overline{B_{p s}(t)}$ and $\overline{T_{i, e}(t)}$ (for all channels $i$ ) are calculated over $N_{\text {seg }}$ time steps with sampling time $d t$, limited by the video bandwidth $B_{v}$ as reported in Appendix B. An average over $N_{\text {seg }}=2400$ is calculated. $L_{1, i}$ and $L_{2, i}$ are calculated for all channels $i$ using

$$
\begin{aligned}
L_{1, i}(t)= & \sum_{j=1}^{N_{\text {seg }}}\left\{\left(T_{i, e}\left(t+\left(j-N_{\text {seg }}\right) d t\right)-\overline{T_{i, e}\left(t+\left(j-N_{\text {seg }}\right) d t\right)}\right)\right. \\
& \left.\left(B_{\text {or }}\left(t+\left(j-N_{\text {seg }}\right) d t\right)-\overline{B_{\text {or }}\left(t+\left(j-N_{\text {seg }}\right) d t\right)}\right)\right\} \\
L_{2, i}(t)= & \sum_{j=1}^{N_{\text {seg }}}\left\{\left(T_{i, e}\left(t+\left(j-N_{\text {seg }}\right) d t\right)-\overline{T_{i, e}\left(t+\left(j-N_{\text {seg }}\right) d t\right)}\right)\right. \\
& \left.\left(B_{\text {ps }}\left(t+\left(j-N_{\text {seg }}\right) d t\right)-\overline{B_{\text {ps }}\left(t+\left(j-N_{\text {seg }}\right) d t\right)}\right)\right\}
\end{aligned}
$$

The phase between $L_{1, i}$ and $L_{2, i}$ is calculated using $\arctan \left(\frac{L_{2, i}}{L_{1, i}}\right)$, where the signs of $L_{1, i}$ and $L_{2, i}$ are taken into account to arrive at a phase between 0 and $2 \pi$. The phase difference between adjacent channels is calculated and it is checked whether the differences are within $0.75 \pi$ of $\pi$, using

$$
\| \arctan \left(\frac{L_{2, i+1}}{L_{1, i+1}}\right)-\arctan \left(\frac{L_{2, i}}{L_{1, i}}\right)|-\pi|<0.75 \pi
$$

for every $i=2$ to $N_{c h}-2$. Detection of $2 / 1$ NTMs is possible using $0.25 \pi$, but detection of $3 / 2$ NTMs required an antiphase bound of $0.75 \pi$, which is larger than the value of $0.25 \pi$ used by Reich et al.[16] Channel 1 and channel $N_{c h}$ are omitted, because it cannot be checked whether these channels show increasing first derivatives. If an anti-correlation is found, the index $j$ is chosen such that $A_{j}$ is the smallest of both 
amplitudes $A_{i}=L_{1, i}^{2}+L_{2, i}^{2}$ and $A_{i+1}=L_{1, i+1}^{2}+L_{2, i+1}^{2}$. For all the phase differences that show an anti-correlation, it is checked whether the amplitude shows an increasing first derivative by demanding that $A_{j}-A_{j-1}<A_{j+1}-A_{j}$.

If at this stage one or multiple $A_{j}$ values associated with anti-correlations are found, the point with the highest value of the correlation amplitude $A_{j-1}+A_{j}+A_{j+1}$ is used. If no minima is found, the previously determined location is maintained. The related position of the mode is expressed in terms of the ECE frequency $f_{E C E, j}$, which is the centre ECE frequency of channel $j$.

\section{References}

[1] M. Shimada, D.J. Campbell, V. Mukhovatov, M. Fujiwara, N. Kirneva, K. Lackner, M. Nagami, V.D. Pustovitov, N. Uckan, J. Wesley, N. Asakura, A.E. Costley, A.J.H. Donné, E.J. Doyle, A. Fasoli, C. Gormezano, Y. Gribov, O. Gruber, T.C. Hender, W. Houlberg, S. Ide, Y. Kamada, A. Leonard, B. Lipschultz, A. Loarte, K. Miyamoto, V. Mukhovatov, T.H. Osborne, A. Polevoi, and A.C.C. Sips. Progress in the ITER Physics Basis Chapter 1: Overview and summary. Nuclear Fusion, 47(6):S1, 2007.

[2] R. J. La Haye. Neoclassical tearing modes and their control. Physics of Plasmas, 13(5):055501, 2006.

[3] R. Prater. Heating and current drive by electron cyclotron waves. Physics of Plasmas, 11(5):2349-2376, 2004.

[4] O Sauter, M A Henderson, G Ramponi, H Zohm, and C Zucca. On the requirements to control neoclassical tearing modes in burning plasmas. Plasma Physics and Controlled Fusion, 52(2):025002, 2010.

[5] R.J. La Haye, J.R. Ferron, D.A. Humphreys, T.C. Luce, C.C. Petty, R. Prater, E.J. Strait, and A.S. Welander. Requirements for alignment of electron cyclotron current drive for neoclassical tearing mode stabilization in iter. Nuclear Fusion, 48(5):054004, 2008.

[6] $\mathrm{H}$ van den Brand, M R de Baar, N J Lopes Cardozo, and E Westerhof. Integrated modelling of island growth, stabilization and mode locking: consequences for NTM control on ITER. Plasma Physics and Controlled Fusion, 54(9):094003, 2012.

[7] Q. Yu and S. Günter. Locking of neoclassical tearing modes by error fields and its stabilization by RF current. Nuclear Fusion, 48(6):065004, 2008.

[8] M.E. Austin, P.E. Phillips, W.L. Rowan, J. Beno, H-P. Liu, A. Ouroua, R.F. Ellis, R.W. Harvey, A.E. Hubbard, G. Taylor and D.W. Johnson. ITER ECE: Plans and Challenges. In $E C-15$ Joint workshop on ECE and ECRH, page P2.170, 2008.

[9] M. Bornatici, R. Cano, O. De Barbieri, and F. Engelmann. Electron cyclotron emission and absorption in fusion plasmas. Nuclear Fusion, 23(9):1153, 1983.

[10] Richard Fitzpatrick. Helical temperature perturbations associated with tearing modes in tokamak plasmas. Physics of Plasmas, 2(3):825-838, 1995.

[11] M. Reich, K. Behler, A. Bock, L. Giannone, M. Lochbrunner, M. Maraschek, E. Poli, C. Rapson, J. Stober, W. Treutterer and ASDEX Upgrade team. ECCD based NTM control at ASDEX Upgrade. In 39th EPS Conference 61 16th Int. Congress on Plasma Physics, page P1.076, 2012.

[12] M. Reich, L. Barrera-Orte, K. Behler, A. Bock, L. Giannone, M. Maraschek, E. Poli, C. Rapson, J. Stober, W. Treutterer and ASDEX Upgrade team. First results of closed loop feedback control of NTMs at ASDEX Upgrade. In 39th EPS Conference 83 16th Int. Congress on Plasma Physics, page PD4.004, 2012.

[13] A. Manini, J. Berrino, S. Cirant, G. D'Antona, F. Gandini, G. Grünwald, F. Leuterer, M. Maraschek, F. Monaco, G. Neu, G. Raupp, D. Sormani, J. Stober, W. Suttrop, W. Treutterer, D. Wagner, and H. Zohm. Development of a feedback system to control MHD instabilities in ASDEX Upgrade. Fusion Engineering and Design, 82(5-14):995 - 1001, 2007. Proceedings of the 24th Symposium on Fusion Technology - SOFT-24.

[14] E. Westerhof, E. Farshi, J.A. Hoekzema, W. Bongers, O.G. Kruijt, J.W. Oosterbeek and J. Scholten. A GENERIC METHOD FOR CONTROLLED ECRH/ECCD LOCALISATION. In EC-13 Joint workshop on ECE and ECRH, page 357, Nizhny Novgorod, Russia, 2004.

[15] J. Berrino, E. Lazzaro, S. Cirant, G. D'Antona, F. Gandini, E. Minardi, and G. Granucci. Electron cyclotron emission temperature fluctuations associated with magnetic islands and real-time identification and control system. Nuclear Fusion, 45(11):1350, 2005.

[16] M. Reich, A. Bock, M. Maraschek, and ASDEX Upgrade Team. NTM LOCALIZATION 
BY CORRELATION OF T-e AND dB/dt. FUSION SCIENCE AND TECHNOLOGY, 61(4):309-313, MAY 2012.

[17] J.A. Snipes, D. Beltran, T. Casper, Y. Gribov, A. Isayama, J. Lister, S. Simrock, G. Vayakis, A. Winter, Y. Yang, and L. Zabeo. Actuator and diagnostic requirements of the ITER Plasma Control System. Fusion Engineering and Design, (0):-, 2012.

[18] R.J. La Haye, R. Prater, R.J. Buttery, N. Hayashi, A. Isayama, M.E. Maraschek, L. Urso, and H. Zohm. Cross-machine benchmarking for ITER of neoclassical tearing mode stabilization by electron cyclotron current drive. Nuclear Fusion, 46(4):451, 2006.

[19] R.M.J. Sillen, M.A.F. Allaart, W.J. Goedheer and A. Kattenberg. NOTEC: A CODE TO SIMULATE ELECTRON CYCLOTRON EMISSION SPECTRA OF PLASMAS WHICH INCLUDE NON-THERMAL POPULATIONS. Rijnhuizen Report 86-165, FOM Institute for Plasma Physics, Nieuwegein, The Netherlands, 1987.

[20] D De Lazzari and E Westerhof. The role of asymmetries in the growth and suppression of neoclassical tearing modes. Plasma Physics and Controlled Fusion, 53(3):035020, 2011.

[21] H J Hartfuss, T Geist, and M Hirsch. Heterodyne methods in millimetre wave plasma diagnostics with applications to ECE, interferometry and reflectometry. Plasma Physics and Controlled Fusion, 39(11):1693, 1997.

[22] B A Hennen, E Westerhof, P W J M Nuij, J W Oosterbeek, M R de Baar, W A Bongers, A Bürger, D J Thoen, M Steinbuch, and the TEXTOR Team. Real-time control of tearing modes using a line-of-sight electron cyclotron emission diagnostic. Plasma Physics and Controlled Fusion, 52(10):104006, 2010.

[23] A. Isayama, Y. Kamada, N. Hayashi, T. Suzuki, T. Oikawa, T. Fujita, T. Fukuda, S. Ide, H. Takenaga, K. Ushigusa, T. Ozeki, Y. Ikeda, N. Umeda, H. Yamada, M. Isobe, Y. Narushima, K. Ikeda, S. Sakakibara, K. Yamazaki, K. Nagasaki, and the JT-60 Team. Achievement of high fusion triple product, steady-state sustainment and real-time NTM stabilization in high- $\beta_{p}$ p ELMy H-mode discharges in JT-60U. Nuclear Fusion, 43(10):1272, 2003.

[24] Jinhong Yang, Qingquan Yu, Sizheng Zhu, and G. Zhuang. Electron temperature difference between the o-point and x-point of a magnetic island. Physics of Plasmas, 16(9):092308, 2009.

[25] J P Meskat, H Zohm, G Gantenbein, S Günter, M Maraschek, W Suttrop, Q Yu, and ASDEX Upgrade Team. Analysis of the structure of neoclassical tearing modes in ASDEX Upgrade. Plasma Physics and Controlled Fusion, 43(10):1325, 2001.

[26] V.S. Udintsev, B.Ph. van Milligen, F.C. Schüller, A. Krämer-Flecken, A.J.H. Donné, J.C. van Gorkom, C.W. Domier, and the TEXTOR-team. Plasma transport properties in the presence of MHD modes studied by ECE at TEXTOR. Nuclear Fusion, 43(11):1424, 2003.

[27] L. Urso, H. Zohm, A. Isayama, M. Maraschek, E. Poli, ASDEX Upgrade Team, and JT-60 Team. ASDEX Upgrade-JT-60U comparison and ECRH power requirements for NTM stabilization in ITER. Nuclear Fusion, 50(2):025010, 2010.

[28] M. E. Austin. Resolution of Electron Cyclotron Emission Measurements of Magnetohydrodynamic Modes in ITER. Fusion Science and Technology, 59(4):647-650, MAY 2011. 16th Joint Workshop on Electron Cyclotron Emission and Electron Cyclotron Resonance Heating (EC-16), Inst Plasma Phys Chinese Acad Sci, Sanya, People's Republic of China, April 12-15, 2010.

[29] A.R. Polevoi, S.Y. Medvedev, V.S. Mukhovatov, A.S. Kukushkin, Y. Murakami, M. Shimada, and A.A. Ivanov. ITER Confinement and Stability Modelling. Journal of Plasma and Fusion Research SERIES, 5:82-87, 2002.

[30] A.A. Balakin, M.A. Balakina, and E. Westerhof. ECRH power deposition from a quasi-optical point of view. Nuclear Fusion, 48(6):065003, 2008.

[31] Charles L. Philips, John M. Parr, and Eve A. Riskin. Signals, Systems and Transforms. Prentice Hall, Upper Saddle River (NY), third edition, 2003.

[32] S Cirant, J Berrino, F Gandini, G Granucci, F Iannone, E Lazzaro, G D’Antona, D Farina, K Koppenburg, S Nowak, and G Ramponi. Crucial issues of multi-beam feed-back control with ECH/ECCD in fusion plasmas. Journal of Physics: Conference Series, 25(1):223, 2005.

[33] G D Conway. Turbulence measurements in fusion plasmas. Plasma Physics and Controlled Fusion, 50(12):124026, 2008.

[34] M. van Berkel, G. Witvoet, M.R. de Baar, P.W.J.M. Nuij, H.G. ter Morsche, and M. Steinbuch. Real-time wavelet detection of crashes in limit cycles of non-stationary fusion plasmas. Fusion Engineering and Design, 86(12):2908 - 2919, 2011.

[35] W. A. Bongers, V. van Beveren, D. J. Thoen, P. J. W. M. Nuij, M. R. de Baar, A. J. H. Donné, E. Westerhof, A. P. H. Goede, B. Krijger, M. A. van den Berg, M. Kantor, M. F. Graswinckel, 
B. A. Hennen, and F. C. Schüller. Intermediate frequency band digitized high dynamic range radiometer system for plasma diagnostics and real-time tokamak control. Review of Scientific Instruments, 82(6):063508, 2011.

[36] A. Collazos, R. Bertizzolo, R. Chavan, F. Dolizy, F. Felici, T.P. Goodman, M.A. Henderson, J.D. Landis, and F. Sanchez. Progress on the ITER H\&CD EC Upper Launcher Steering-Mirror Control System. Plasma Science, IEEE Transactions on, 38(3):441 -447, 2010.

[37] W. A. Bongers, A. P. H. Goede, E. Westerhof, J. W. Oosterbeek, N. J. Doelman, F. C. Schüller, M. R. De Baar, W. Kasparek, W. Wubie, D. Wagner, J. Stober, and TEXTOR Team. Magnetic island localization for NTM control by ECE viewed along the same optical path of the ECCD beam. Fusion Science and Technology, 55(2):188-203, FEB 2009.

[38] I.H. Hutchinson. Principles of Plasma Diagnostics. Cambridge University Press, Cambridge, United Kingdom, Second edition, 2002.

[39] D. B. Batchelor, R. C. Goldfinger, and H. Weitzner. Ray Tracing near the Electron Cyclotron Frequency with Application to EBT. Plasma Science, IEEE Transactions on, 8(2):78 -89, june 1980.

[40] R. Prater, D. Farina, Yu. Gribov, R.W. Harvey, A.K. Ram, Y.-R. Lin-Liu, E. Poli, A.P. Smirnov, F. Volpe, E. Westerhof, A. Zvonkov, and the ITPA Steady State Operation Topical Group. Benchmarking of codes for electron cyclotron heating and electron cyclotron current drive under ITER conditions. Nuclear Fusion, 48(3):035006, 2008.

[41] Kritz A.H. and Hsuan H. and Goldfinger R.C. and Batchelor D.C. In 3rd Int. Symp. on Heating in Toroidal Plasmas ECE, volume 2, page 707, Brussels, Belgium, 1982.

[42] E. Westerhof. Implementation of TORAY at JET. National Technical Information Service document no PB91-114819INZ, Rijnhuizen Report 89-183, FOM Institute for Plasma Physics, Nieuwegein, The Netherlands, 1989. (Copies may be ordered from the National Technical Information Service, Springfield, VA 22161, USA). 